\title{
The Prevalence and Control of Type 2 Diabetes Mellitus in Residents of a Rural Town, Shandong Province, China
}

\author{
Maoti Wei (iD) ${ }^{1, *}$ \\ Li Dong ${ }^{2, *}$ \\ Fenghua Wang' \\ Kai Cui ${ }^{3}$ \\ Delong $\mathrm{Ma}^{2}$ \\ Ning Yang ${ }^{4}$ \\ Yuming $\mathrm{Li}^{4}$ \\ 'Center of Clinical Epidemiology, TEDA \\ International Cardiovascular Hospital, \\ Tianjin, People's Republic of China; \\ ${ }^{2}$ Department of Pediatrics, Center \\ Hospital of Beikuo Town, Rizhao City, \\ Shandong Province, People's Republic of \\ China; ${ }^{3}$ Department of Disease \\ Prevention and Control, Hospital of \\ Gaoxing Town, Rizhao City, Shandong \\ Province, People's Republic of China; \\ ${ }^{4}$ Department of Hypertension, TEDA \\ International Cardiovascular Hospital, \\ Tianjin, People's Republic of China
}

*These authors contributed equally to this work
Correspondence: Ning Yang; Yuming Li Email yangningzxdı@sina.com; cardiolab@live.com
Objective: To understand the prevalence and control of type 2 diabetes mellitus (T2DM) in a rural area, a cross-sectional study was carried out.

Methods: Blood glucose was determined in residents over 60 years during the National Basic Public Health Service project carrying on in a rural town of Shandong province. Combined with detail information of diabetic history, the prevalence rate of this disease was calculated with a cutoff of more than $7.0 \mathrm{mmol} / \mathrm{L}$ of fasting plasma glucose (FPG). Furthermore, according to FPG levels, the control rate of diabetes was evaluated. Basic information and clinical laboratory tests were analyzed with proper methods for univariate analysis. Multivariable logistic analysis was used to explore multiple variables associated with the control effect of T2DM.

Results: The prevalence rate of T2DM was $13.5 \%$ (95\% CI 12.4\% 14.7\%) without age difference in residents over 60 -year old $\left(X^{2}=1.078, P=0.898\right)$. And the rate was higher in the female $(16.1 \%$, 95\% CI $14.4 \% \sim 17.8 \%)$ than that in the male $(11.3 \%, 95 \%$ CI $9.7 \%$ $\sim 12.9 \%$ ). The control rate of diabetes was $44.5 \%$ (95\% CI $39.4-49.6 \%$, cutoff $<6.1 \mathrm{mmol} / \mathrm{L}$ for FPG) and 59.6\% (95\% CI 54.5-64.6\%, cutoff $<7.0 \mathrm{mmol} / \mathrm{L}$ for FPG), respectively. Most T2DM residents had balance foods, and a high proportion of drugs or insulin were used; however, the proportion of keeping exercise and modifying behaviors was low. Nearly $80 \%$ of the diabetic individuals used at least one drug or insulin. However, the more drugs or insulin used, the higher the FPG level was.

Conclusion: A relative high level of T2DM prevailed in the rural area under fast economytransforming. T2DM was controlled poor and measures should be further optimized to achieve ideal effect, especially for the use of anti-diabetic drugs.

Keywords: rural area, type 2 diabetes mellitus, the control of diabetes

Type 2 diabetes mellitus (T2DM) is a major global health problem causing significant morbidity and mortality, and the International Diabetes Federation (IDF) estimates that there will be 578 million adults with diabetes by 2030 , and 700 million by $2045 .{ }^{1}$ The worldwide prevalence of diabetes mellitus has been increasing over the past few decades, particularly in developing countries. There has been a rapid escalation of T2DM in developing countries, with varied prevalence according to rural vs urban habitat and degree of urbanization. ${ }^{2}$ Real-world evidence of patient profiles and diabetes care practices in developing countries results showed that glycemic control in individuals with T2DM remained suboptimal over a 12-year period. ${ }^{3}$ In China, the number of T2DM patients is expected to increase from 20.8 million (in 2000) to 
42.3 million by $2030 .{ }^{4}$ Since "the outline of healthy China in 2030" plan issued on October 25, 2016, ${ }^{5}$ the Chinese government had put great efforts to ensure the realization of the aims and the improvements of health conditions for residents in the new era.

Among the government efforts, fighting against chronic diseases, especially T2DM, was put into the first place because of its fast spreading. China listed at the top of developing countries and the trend remained unchanged from 1980 to 2014, and the global share of adults with diabetes who live in China increased, by contrast with the USA, where the share decreased. ${ }^{6}$ The living standard of the population has also been rising in accordance with the development of Chinese economy. Companied with the improvement of living standards was the rapid increase of T2DM. ${ }^{4}$ Data showed that the prevalence of T2DM in people aged 18 and above was $11.2 \%$ during 2015 to 2017 which increased more than 15 times compared with data of 1980. There was also a trend of narrowing the T2DM prevalence gap between the urban and the rural. So, in order to solve health problems in China, the rural residents and T2DM must be focused on in the first place.

According to the results of large-scale epidemiological investigations, the awareness rate, the treatment rate and the control rate of T2DM in China have improved in recent years, but they are still at a low level, especially in rural areas. With the development of national basic public health services, the primary medical institutions had conducted the services, and a large number of data related to residents' health condition were collected. In this paper, the area where the population lived in is a rural town in Shandong province, and this town is experiencing transforming of living conditions from the countryside towards the city. Therefore, making full use of these data would further clarify the epidemic status of T2DM and assess the effect of disease prevention and control in rural areas, which will also provide guidance for further developing better health services.

\section{Materials and Methods}

\section{Subjects and Information Collection}

From February 2020 to December 2020, relying on the national basic public health service project, a township hospital which covers primary health care carried out health examinations for residents over 60 years old in Shandong province. The checkup collected 132 items including basic information, physical examinations, blood biochemistry tests, ECG data, personal habits, disease histories, treatments, and psychological status of the participants, and informed the participants that the data may be used for scientific research and obtained a personal data use license with a signed consent form. For the sake of personal confidentiality, before analysis, the data were first checked and then decrypted. The study was conducted in accordance with the declaration of Helsinki and approved by the Clinical Research Ethics Committee of TEDA International Cardiovascular Hospital.

Fast plasma glucose (FPG) levels were measured, and the history of diabetes and the medication were investigated. The analysis process of the subjects is shown in Figure 1.

\section{Demographic Characteristics}

In this study, T2DM was diagnosed and determined according to the screening process and the key points and methods provided by the national guidelines for the prevention and treatment of diabetes at the population level (2020 Edition). ${ }^{1,4}$ In short, the diagnosis was determined based on the outpatient blood glucose combined with the disease history. If the participants had the fast blood glucose agreeing with the standard of T2DM or were taking anti-diabetes drugs, they were judged with T2DM. BMI was calculated by dividing the weight in kilograms by the height in meters squared.

\section{Definitions}

It was defined based on fast plasma glucose measurement (FPG $\geq 7.0 \mathrm{mmol} / \mathrm{L}$ or previously diagnosed diabetes), which means the current diabetes mellitus should include previously known and newly (undiagnosed) diabetes.

Awareness rate was defined as the proportion of individuals with self-reported physician-diagnosed diabetes among all participants with diabetes. Treatment rate was defined as the percentage of diabetic patients who had taken diabetic medications. The control rate of diabetes was characterized as the proportion of participants with an FPG level among diabetic patients who were treated with diabetic medications and the well-controlled were with FPG $<6.1 \mathrm{mmol} / \mathrm{L}$, the poorer controlled with FPG between $6.1 \mathrm{mmol} / \mathrm{L}$ and $7.0 \mathrm{mmol} / \mathrm{L}$, and the failure of controlled with $\mathrm{FPG} \geq 7.0 \mathrm{mmol} / \mathrm{L}$.

\section{Data Processing and Statistical Methods}

After establishing the database, data were cleaned for unqualified data before analysis. Quantitative data were described using mean \pm standard deviation or median with quartile percentiles. Student's $t$-test (or $t^{\prime}$-test) or 


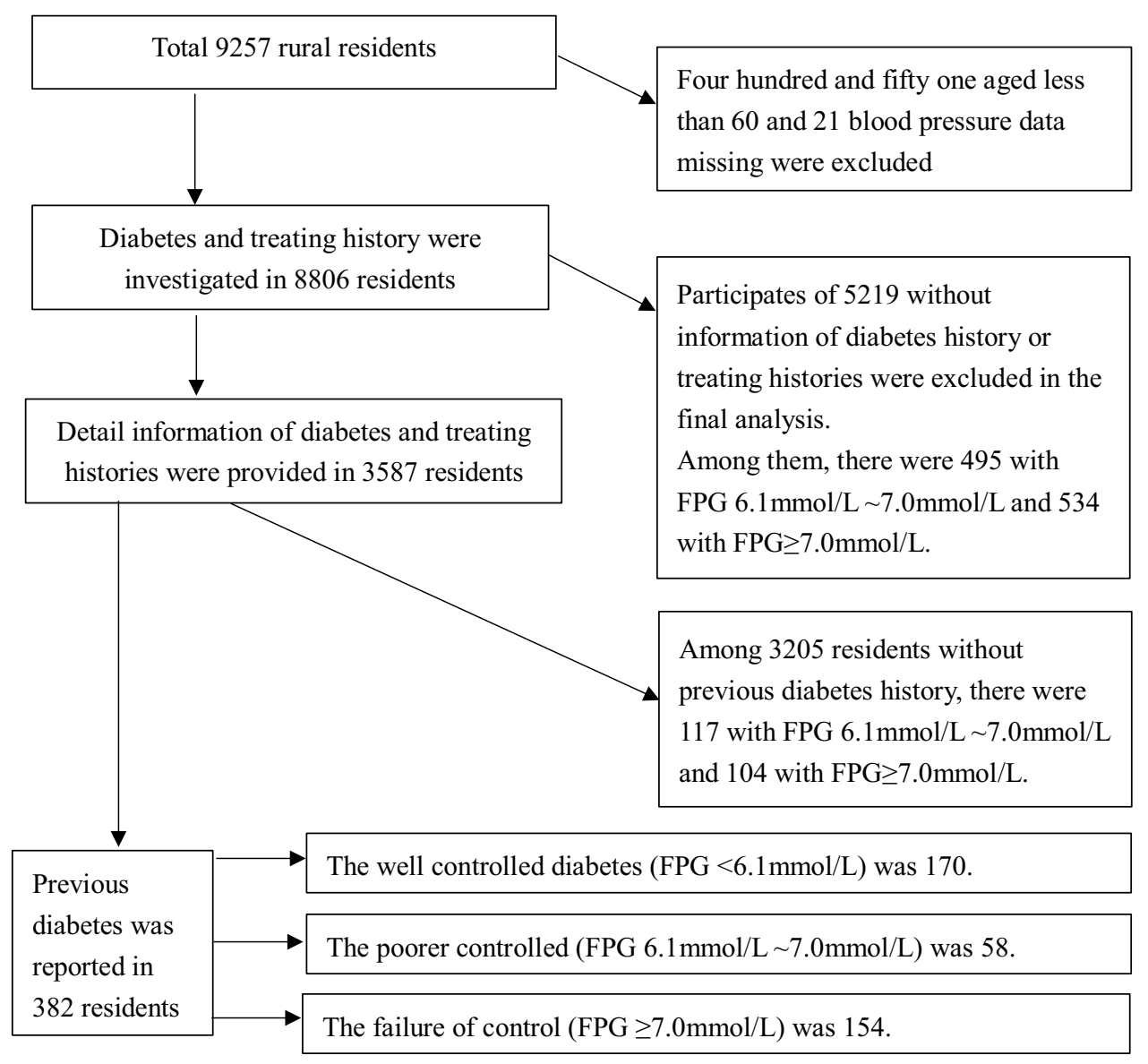

Figure I Data process flow for investigating the prevalence of diabetes in a rural area in Shandong province, China.

one-way ANOVA were used for the comparison between groups, and SNK (or LSD) was used for multiple comparisons among groups for post hoc analysis. Qualitative data (or counting data) were described as percentage or proportions, and comparison between groups was carried out using chi-square test. Tests of two sides and $P<0.05$ were statistically significant. Multivariable logistic regression analysis was used to analyze the factors related to diabetes control. In the regression, the variable screening method was backward: conditional, the probability of a variable entering the equation was 0.05 , excluding 0.10 , and the other parameters were system default. The research data analysis and processing was completed by professional statistical analysts using SPSS software version 26.00 (Armonk, NY: IBM Corp).

\section{Results}

According to FPG results or disease history, the rate of diabetes was $13.5 \%((382+104) / 3587,95 \%$ CI $12.4 \%$ $\sim 14.7 \%$ ) (The standardized age-sex prevalence rate was also $13.5 \%$ based on the standard population of the World
Health Organization $\left.{ }^{7}\right)$ and there was no difference among age groups over 60 years old $\left(X^{2}=1.078, P=0.898\right)$ (Figure 2A). However, the rate was $11.3 \%(171 / 1516,95 \%$ CI $9.7 \%-12.9 \%)$ in men, lower than $16.1 \%(315 / 1957,95 \%$ CI $14.4 \%-17.8 \%)$ in women $\left(X^{2}=16.465, P=0.000\right)$. Also, differences among age groups were observed between men and women (stratified $X^{2}=16.003, P=0.000$ ) (Figure 2B).

Among the patients with clear diabetic histories, the well-controlled rate of diabetes $(\mathrm{FPG}<6.1 \mathrm{mmol} / \mathrm{L})$ was $44.5 \%(170 / 382,95 \%$ CI $39.4 \%-49.6 \%)$, the poorer controlled rate $(\mathrm{FPG}<7.0 \mathrm{mmol} / \mathrm{L})$ was $59.6 \%((170+58) / 382$, 95\% CI $54.5 \%-64.6 \%$ ). No different distributions of demographic characteristics (gender and age), habitats (smoke history and alcohol history), physical measurements (waist circumference, BMI, systolic pressure and diastolic pressure) and blood lipid indexes (total cholesterol, triglyceride, LDL cholesterol, HDL cholesterol) were observed among diabetic patients (Table 1).

According to the Chinese guideline for treating diabetes, a comprehensive strategy was used including diabetes education, an emphasis on life style modification, 

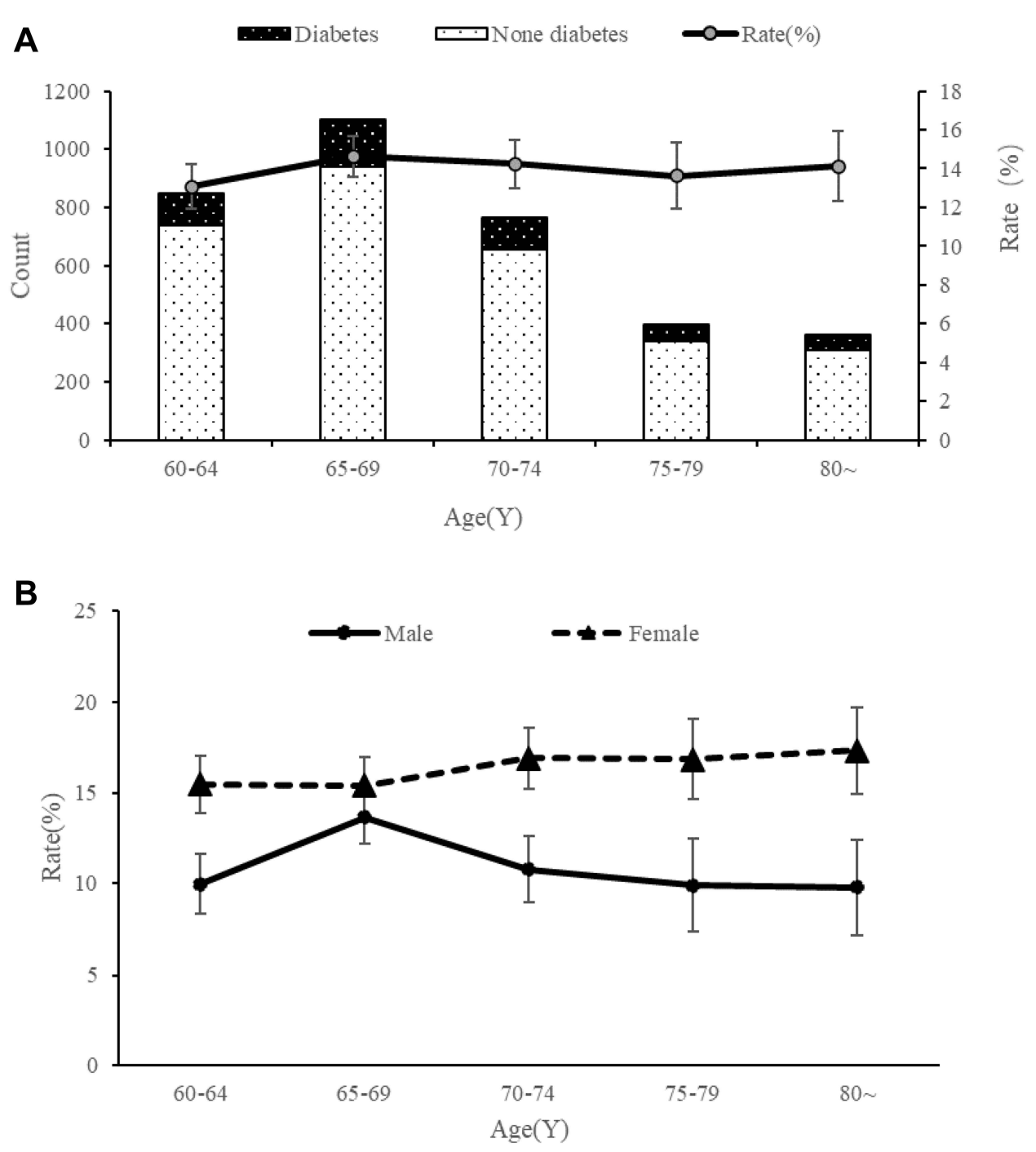

Figure 2 Distribution of diabetes mellitus among people over 60 years old in a rural area, Shandong province, China.

maintain an adequate diet and physical activity, glycemic control using drugs and follow a specific program of periodic medical checks, which was analogized as "one carriage with five horses". Among 382 patients, nearly $80 \%$ (299/382, 95\% 73.8-82.3\%) used as least one drug or insulin. The most commonly used drugs were metformin (usage rate was $82.9 \%, 248 / 299$ ), glipizide (usage rate was $29.1 \%, 87 / 299$ ) and insulin (usage rate was $10.7 \%$, 32/299) (Table 2, Figure 3).

Demographic characteristics (gender, age, smoking, drinking, balance nutrition, exercises) and domain medicines for diabetes control and interactions (metformin, glipizide, insulin, metformin*insulin, metformin*glipizide, glipizide*insulin) were included in multivariable analysis. Results showed that the usages of metformin, glipizide and insulin were not related to the control of FBG, on the contrary, higher levels of FBG in antidiabetic users were observed. However, the combination of anti-diabetic medicines may reduce fasting blood glucose (Table 3).

\section{Discussion}

It would be convenient to carry out chronic noncommunicable disease investigation using the data collected by the national public health service project, and the results would be target on the population exactly. ${ }^{6,8}$ Increased by more than 15 times compared with data of 1980, the prevalence of diabetes in people aged 18 and above was $11.2 \%$ during 2015 to 2017 , and there was a decreased trend of prevalence of diabetes between the urban and the rural areas. ${ }^{6}$ In this paper, the area where the 
Table I Demographic Characteristics Associated Blood Glucose in Old Patients of Diabetes in a Rural Area, Shandong Province, China

\begin{tabular}{|c|c|c|c|c|c|}
\hline & \multicolumn{3}{|c|}{ Fast Plasma Glucose (mmol/L) } & \multirow[t]{2}{*}{ Statistics } & \multirow[t]{2}{*}{$\mathbf{P}$} \\
\hline & $<6.1$ & $6.1 \sim 7.0$ & $\geq 7.0$ & & \\
\hline Gender (Male/Female) & $57 / 113$ & $15 / 43$ & $48 / 106$ & $\chi^{2}=1.187$ & 0.552 \\
\hline Age (Year) & $69.9 \pm 6.6$ & $69.6 \pm 6.5$ & $69.6 \pm 6.5$ & $F=0.088$ & 0.916 \\
\hline Smoke history $(\mathrm{Y} / \mathrm{N})$ & $27 / 143$ & $8 / 50$ & $21 / 133$ & $\chi^{2}=0.367$ & 0.832 \\
\hline Alcohol history $(\mathrm{Y} / \mathrm{N})$ & $32 / 138$ & $7 / 51$ & $23 /|3|$ & $\chi^{2}=3.244$ & 0.197 \\
\hline Cardiovascular disease comorbidity $(\mathrm{Y} / \mathrm{N})$ & $119 / 5 \mid$ & $41 / 17$ & $92 / 62$ & $\chi^{2}=4.468$ & 0.107 \\
\hline Waist circumference $(\mathrm{cm})$ & $91.7 \pm 12.7$ & $91.6 \pm 13.4$ & $90.3 \pm 11.7$ & $F=0.603$ & 0.548 \\
\hline BMI $\left(\mathrm{kg} / \mathrm{m}^{2}\right)$ & $25.5 \pm 3.3$ & $25.4 \pm 3.8$ & $25.1 \pm 3.8$ & $F=0.452$ & 0.637 \\
\hline Systolic pressure $(\mathrm{mmHg})$ & $|52.6 \pm 2| . \mid$ & $150.8 \pm 19.8$ & $152.7 \pm 22.7$ & $F=0.187$ & 0.830 \\
\hline Diastolic pressure $(\mathrm{mmHg})$ & $85.2 \pm 10.8$ & $85.3 \pm 11.9$ & $84.0 \pm 11.6$ & $F=0.596$ & 0.552 \\
\hline Total cholesterol (mmol/L) & $5.6 \pm 1.4$ & $5.5 \pm 1.0$ & $5.6 \pm 1.1$ & $F=0.388$ & 0.679 \\
\hline Triglyceride (mmol/L) & $1.5 \pm 0.9$ & $1.8 \pm 1.3$ & $1.8 \pm 1.7$ & $F=2.029$ & 0.133 \\
\hline LDL cholesterol (mmol/L) & $2.6 \pm 0.9$ & $2.5 \pm 0.7$ & $2.6 \pm 0.7$ & $F=0.586$ & 0.557 \\
\hline HDL cholesterol (mmol/L) & $1.7 \pm 0.5$ & $1.6 \pm 0.5$ & $1.6 \pm 0.5$ & $F=0.485$ & 0.616 \\
\hline
\end{tabular}

Abbreviations: BMI, body mass index; LDL, low-density lipoprotein; HDL, high-density lipoprotein.

Table 2 Treating Measures Used in Old Patients of Diabetes in a Rural Area, Shandong Province, China

\begin{tabular}{|c|c|c|c|c|c|}
\hline & \multicolumn{3}{|c|}{ Fast Plasma Glucose (mmol/L) } & \multirow[t]{2}{*}{ Statistics } & \multirow[t]{2}{*}{$P$} \\
\hline & $<6.1$ & $6.1 \sim 7.0$ & $\geq 7.0$ & & \\
\hline Maintain a balance diet $(\mathrm{Y} / \mathrm{N}) *$ & $167 / 3$ & $58 / 0$ & $152 / 2$ & Fisher & 0.851 \\
\hline Physical activity $(\mathrm{Y} / \mathrm{N})$ & $1 / 169$ & I/ 57 & $6 / 148$ & Fisher & 0.101 \\
\hline Drugs or insulin $(\mathrm{Y} / \mathrm{N})$ & $109 / 61$ & $50 / 8$ & $140 / 14$ & $\chi^{2}=36.634$ & $<0.001$ \\
\hline Number of drugs or insulin & & & & $\chi^{2}=47.336$ & $<0.001$ \\
\hline 0 & 61 & 8 & 14 & & \\
\hline I & 86 & 33 & 79 & & \\
\hline 2 & 20 & 16 & 57 & & \\
\hline 3 & 3 & I & 4 & & \\
\hline Metformin $(\mathrm{Y} / \mathrm{N})$ & $93 / 77$ & $44 / 14$ & $111 / 43$ & $\chi^{2}=14.302$ & $<0.001$ \\
\hline Glipizide (Y/N) & $21 / 149$ & $|7 / 4|$ & $49 / 105$ & $\chi^{2}=19.068$ & $<0.001$ \\
\hline Insulin $(\mathrm{Y} / \mathrm{N})$ & $4 / 166$ & $3 / 55$ & $25 / 129$ & $\chi^{2}=21.199$ & $<0.001$ \\
\hline
\end{tabular}

Note: *Balance diet and physical activity were self-reported.

population is located is a classic economy-transforming area and the living standards transform from the level of countryside towards that of the city. The process of urbanization in this area has accelerated significantly, and the population in this area has gradually changed from farmers to urban residents. Under such conditions, a rapid increase in the prevalence of diabetes could happen. At the same time, with the aging of society, the proportion of the elderly over 60 years in China has increased year by year, from $10 \%$ in $2000,12 \%$ in 2008 and $17.3 \%$ in $2017 .^{9}$ With the popularization of new rural cooperative medical system in China, life expectancy of the elderly was also prolonged, and the chronic non-communicable diseases including diabetes would also increase gradually. ${ }^{10}$

The population included in this study is the elderly over 60 years old, accounting for about $17 \%$ of the town's population, which is close to the proportion of China's elderly population in 2019. ${ }^{9}$ From 2007 to 2017 , the prevalence of diabetes in elderly people over 60 was close to or over $20 \%{ }^{4}$ The results showed that the prevalence of diabetes in this area was $13.5 \%$ (95\% CI $12.4 \%$ 


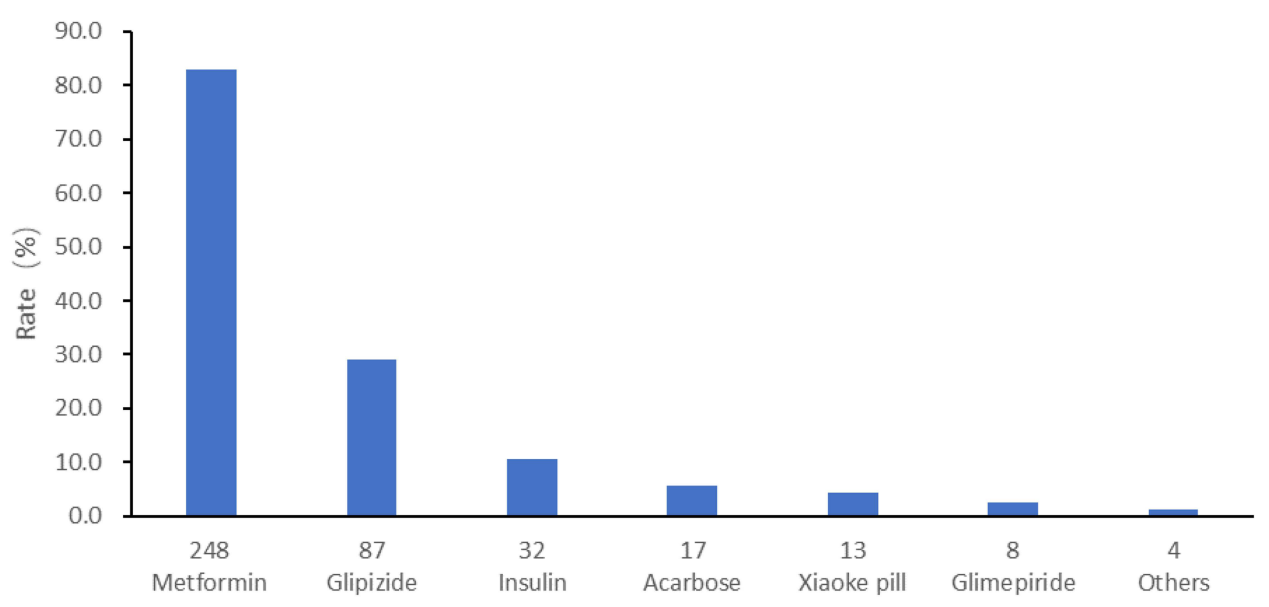

Figure 3 Anti-diabetic drugs were used for old patients of diabetes in a rural area, Shandong province, China.

$\sim 14.7 \%$ ). Compared with previous results, the prevalence of diabetes in this area was relatively low, which may be explained by a higher proportion of undiagnosed diabetes. The population of 8806 was included in this study and the majority of the basic information was not provided, which means that most local residents were not interested in or paid no attention to the diagnosis and treatment of diabetes. Also, to those without providing basic information, the prevalence of diabetes was $10.2 \%$ (534/5219). Therefore, when implementing the national basic public health service project, we must vigorously publicize it so as to continuously improve the awareness rate of the population.

There is no trend of the prevalence of diabetes increasing with age in the population, and this trend is different from the prevalence of type 2 diabetes in Germany, whose model rose steeply from age 50 to age $80 .{ }^{11}$ Also, there was a significant difference between genders in different age groups. The prevalence of diabetes in women was higher than that in men $\left(X^{2}=16.465, P=0.000\right)$ which were manifested in all age groups over 60 years old (stratified chi square $X^{2}=16.003$, $P=0.000$ ) and these results were consistent with those of Xu. ${ }^{10}$

The glycemic control results were investigated according to fasting blood glucose concentration. When the FBG was below $6.1 \mathrm{mmol} / \mathrm{L}$ as the well-controlled standard, the glycemic control rate of the diabetic population in the area was $44.5 \%$. If the glycemic control value below $7 \mathrm{mmol} / \mathrm{L}$ was used as the control standard, the glycemic control rate of the diabetic population in this area was $59.6 \%$. And the results of this study were slightly higher than those of other studies. ${ }^{10}$

According to the Chinese guideline for treating diabetes, a comprehensive strategy was used including diabetes education, an emphasis on life style modification, maintain an adequate diet and physical activity, glycemic control using drugs and follow a specific program of periodic medical checks, which was analogized as "one carriage with five horses". In controlling FBG, the effect of "one carriage with five horses" may not be further investigated. Factors that may affect glycemic control were explored in diabetic patients and the results showed

Table 3 Multivariable Analysis of Factors Associated Blood Glucose in Old Patients of Diabetes in a Rural Area, Shandong Province, China*

\begin{tabular}{|l|c|c|c|l|}
\hline \multirow{2}{*}{} & \multicolumn{2}{|c|}{ Model I (<6.I mmol/L) } & \multicolumn{2}{c|}{ Model 2 (<7.0 mmol/L) } \\
\cline { 2 - 5 } & $\boldsymbol{P}$ & OR (95\% CI) & $P$ & OR (95\% CI) \\
\hline Metformin (Yes) & $<0.001$ & $2.704(1.663-4.339)$ & 0.002 & $2.250(1.343-3.768)$ \\
Glipizide (Yes) & $<0.001$ & $3.352(1.893-5.938)$ & 0.001 & $2.419(1.45 I-4.035)$ \\
Insulin ((Yes) & $<0.001$ & $17.165(4.899-60.140)$ & $<0.00 I$ & $11.051(4.366-27.969)$ \\
Insulin * glipizide & 0.043 & $0.042(0.002-0.908)$ & & \\
\hline
\end{tabular}

Notes: *Multivariable program and parameters used in this study. LOGISTIC REGRESSION VARIABLES Model I (Model 2). /METHOD=BSTEP(COND) gender, age, smoke, drink, healthy diet, exercises, metformin, glipizide, insulin, metformin* insulin, metformin* glipizide, glipizide* insulin. /PRINT=CI(95). /CONTRAST (male)=Indicator (Female). /CONTRAST (Exercise)=Indicator (no exercise). /CONTRAST (No smoke)=Indicator (Smoke). /CONTRAST (Healthy diet)=Indicator (no Healthy diet). / CONTRAST (No drink)=Indicator (Drink). /CRITERIA=PIN (0.05) POUT (0.10) ITERATE (20) CUT (0.5). 
that there were no distribution differences among demographic characteristics, habits, nutritional balance and exercises; however, the usage of anti-diabetic drugs distributed differently. In this area, $78.3 \%$ of the patients used at least one control measure, such as drugs or insulin, and this number was significantly higher than that in $2010 .{ }^{10}$ Data showed that only $25.8 \%$ of the diabetic patients were treated in 2010 in China and this percentage should be improved according to the guideline for prevention and treatment of type 2 diabetes in China (2020 Edition). ${ }^{4}$

Of the patients in controlling diabetes, nearly $1 / 3$ used two more drugs, or drug and insulin combination. However, the effect of using drugs or combination with insulin were not as expected. The results showed that the more drugs or measures were used, the higher the fasting blood glucose level was observed, which was different from conventional knowledge. And this phenomenon was also observed in other populations (personal communication). It is generally believed that the more drugs or control measures are used, the better the control effect of diabetes will be, but this study draws the opposite conclusion. The reason may be that patients strengthen their blood glucose monitoring and adjusted drugs or measures by themselves. Under such conditions, although the use of diabetes control drugs or measures, and the effects of it may not reach the control target. Another explanation would be that diabetic patients often suffered from other chronic diseases and needed to take anti-chronic diseases drugs. Patients with diabetes, especially those in rural areas, may feel that they are taking too many drugs and artificially reduce dosage or drugs or measures for controlling diabetes.

Limitations: This study was based on FPG for diabetes prevalence rate and control effect study. Underestimation of diabetes prevalence may be caused by failure to incorporate a more stable index of glycated hemoglobin (HbA1c). ${ }^{10}$ Even if HbA1c was used as a glycemic control marker, the diagnosis of diabetes may be underestimated in field investigation. ${ }^{1}$ Although only FBG were used in this study, and it was convenient in field screening and consistent with the whole population in this area, so it could also explain the prevention and control of diabetes in rural areas at a certain level.

\section{Conclusion}

In conclusion, the prevalence of diabetes and control situation investigation were carried out based on the FBG collection and information survey from the rural area. Although the prevalence rate of diabetes in the elderly in rural areas with fast economy-transforming was lower than the national level, a relative highly level prevalence of diabetes was observed in this area. Diabetes is poorly controlled and the measures should be further optimized to achieve ideal effect, especially for the use of antidiabetic drugs.

\section{Acknowledgments}

This study was supported by the China International Medical Foundation (grant no. Z-2019-42-1908) and the major special support for chronic disease prevention and control of Tianjin Science and Technology Commission (grant no. 16ZXMJSY00130). We thank all staff members for collecting the data. Without their valuable assistance, it would have been impossible to successfully complete this study. Maoti Wei and Li Dong are co-first authors of this study.

\section{Disclosure}

The authors report no conflicts of interest in this work.

\section{References}

1. International Diabetes Federation. IDF Diabetes Atlas. 9th ed. Brussels, Belgium: International Diabetes Federation; 2019.

2. Misra A, Gopalan H, Jayawardena R, et al. Diabetes in developing countries. $J$ Diabetes. 2019;11(7):522-539. doi:10.1111/17530407.12913

3. Aschner P, Gagliardino JJ, Ilkova H, et al. Persistent poor glycaemic control in individuals with type 2 diabetes in developing countries: 12 years of real-world evidence of the International Diabetes Management Practices Study (IDMPS). Diabetologia. 2020;63 (4):711-721. doi:10.1007/s00125-019-05078-3

4. Diabetes Association of Chinese Medical Association. Guideline for prevention and treatment of type 2 diabetes in China (2020 Edition). Chin Diabetes J. 2021;13(4):315-409. doi:10.3760/cma.j.cn11579120210221-00095

5. Xinhua News Agency. The CPC Central Committee and the State Council issued the outline of "healthy China 2030". Available from: http://www.gov.cn/zhengce/2016-10/25/content_5124174.htm. Accessed October 28, 2021

6. NCD Risk Factor Collaboration (NCD-RisC). Worldwide trends in diabetes since 1980: a pooled analysis of 751 population-based studies with 4.4 million participants. Lancet. 2016;387 (10027):1513-1530. doi:10.1016/S0140-6736(16)00618-8

7. World Health Organization. World (WHO 2000-2025) standard. Available from: https://seer.cancer.gov/stdpopulations/world.who. html. Accessed October 28, 2021.

8. Alemu T, Tadesse T, Amogne G. Glycemic control and its determinants among patients with type 2 diabetes mellitus at Menelik II Referral Hospital, Ethiopia. SAGE Open Med. 2021;9:20503121211023000. doi:10.1177/20503121211023000. eCollection.2021

9. National Population Data 2019. National Bureau of Statistics of China. Available from: https://data.stats.gov.cn/english/easyquery. $\mathrm{htm}$ ? $\mathrm{cn}=\mathrm{C} 01$. Accessed October 28, 2021.

10. $\mathrm{Xu} \mathrm{Y,} \mathrm{Wang} \mathrm{L}, \mathrm{He} \mathrm{J}$, et al. Prevalence and control of diabetes in Chinese adults. JAMA. 2013;310(9):948-959. doi:10.1001/ jama.2013.168118.

11. Tamayo T, Brinks R, Hoyer A, et al. The prevalence and incidence of diabetes in Germany. Dtsch Arztebl Int. 2016;113(11):177-182. 


\section{Publish your work in this journal}

Diabetes, Metabolic Syndrome and Obesity: Targets and Therapy is an international, peer-reviewed open-access journal committed to the rapid publication of the latest laboratory and clinical findings in the fields of diabetes, metabolic syndrome and obesity research. Original research, review, case reports, hypothesis formation, expert opinion and commentaries are all considered for publication. The manuscript management system is completely online and includes a very quick and fair peer-review system, which is all easy to use. Visit http://www.dovepress.com/testimonials.php to read real quotes from published authors.

Submit your manuscript here: https://www.dovepress.com/diabetes-metabolic-syndrome-and-obesity-targets-and-therapy-journal 